\title{
Anatomy of the Photochemical Reaction: Excited- State Dynamics Reveals the C-H Acidity Mechanism of Methoxy Photo-oxidation on Titania
}

\section{Citation}

Kolesov, Grigory, Dmitry Vinichenko, Georgios A. Tritsaris, Cynthia M. Friend, and Efthimios Kaxiras. 2015. "Anatomy of the Photochemical Reaction: Excited-State Dynamics Reveals the C$\mathrm{H}$ Acidity Mechanism of Methoxy Photo-Oxidation on Titania." The Journal of Physical Chemistry Letters 6 (9): 1624-27. https://doi.org/10.1021/acs.jpclett.5b00429.

\section{Permanent link}

http://nrs.harvard.edu/urn-3:HUL.InstRepos:41384041

\section{Terms of Use}

This article was downloaded from Harvard University's DASH repository, and is made available under the terms and conditions applicable to Other Posted Material, as set forth at http:// nrs.harvard.edu/urn-3:HUL.InstRepos:dash.current.terms-of-use\#LAA

\section{Share Your Story}

The Harvard community has made this article openly available.

Please share how this access benefits you. Submit a story.

\section{Accessibility}




\title{
Anatomy of the Photochemical Reaction: Excited-State Dynamics Reveals the $\mathrm{C}-\mathrm{H}$ Acidity Mechanism of Methoxy Photo-oxidation on Titania
}

\author{
Grigory Kolesov, ${ }^{\dagger, \perp}$ Dmitry Vinichenko, ${ }^{\dagger, \perp}$ Georgios A. Tritsaris, ${ }^{\dagger}$ Cynthia M. Friend, ${ }^{\dagger, \ddagger}$ \\ and Efthimios Kaxiras*, $*, \S$
}

${ }^{\dagger}$ School of Engineering and Applied Sciences, ${ }^{\ddagger}$ Department of Chemistry and Chemical Biology, and ${ }^{\S}$ Department of Physics, Harvard University, Cambridge, Massachusetts 02138, United States

\section{Web-Enhanced Feature S Supporting Information}

ABSTRACT: Light-driven chemical reactions on semiconductor surfaces have potential for addressing energy and pollution needs through efficient chemical synthesis; however, little is known about the time evolution of excited states that determine reaction pathways. Here, we study the photo-oxidation of methoxy $\left(\mathrm{CH}_{3} \mathrm{O}\right)$ derived from methanol on the rutile $\mathrm{TiO}_{2}(110)$ surface using $a b$ initio simulations to create a molecular movie of the process. The movie sequence reveals a wealth of information on the reaction intermediates, time scales, and energetics. The reaction is broken in three stages, described by Lewis structures directly derived from the "hole" wave functions that lead to the concept of "photoinduced $\mathrm{C}-\mathrm{H}$ acidity". The insights gained from this work can be generalized to a set of simple rules that can predict the efficiency of photo-oxidation reactions in reactant-catalyst pairs.

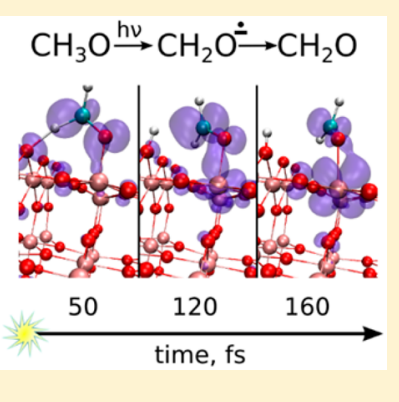

$\mathrm{T}$ here is great interest in the design of materials that can efficiently harness solar energy to drive important chemical processes, including the synthesis of chemicals and fuels and the degradation of environmental pollutants. Generally, the choice of a photocatalyst is guided by thermodynamic considerations, that is, comparison of the energy of charge carriers (electrons and holes) created by photoexcitation to the energetic requirements for a specific reaction. ${ }^{1-4}$ Although this is a necessary condition, it is not sufficient because the evolution of a system on the excited-state potential energy surface can have completely different features than the ground-state thermodynamic properties. Thus, understanding heterogeneous photochemistry requires detailed insight into how the key molecular intermediates interacting with the semiconductor photocatalyst evolve in time after excitation to produce the desired reaction. To achieve this understanding, surface-bound intermediates and the reaction path to products must be identified. Such fundamental insights can provide a generalizable model for guiding the design of photocatalysts for specific reactions. Here, we use ab initio nonadiabatic simulations to understand the microscopic mechanism of a prototypical heterogeneous photoreaction, the oxidation of methoxy to formaldehyde on the rutile $\mathrm{TiO}_{2}(110)$ surface. The results provide unprecedented insight into the process that can be analyzed in simple terms involving bond length and bond angle changes using standard chemical concepts like Lewis structures. The analysis naturally yields a set of rules for choosing optimal reactant-catalyst pairs to enhance efficiency.

Photo-oxidation of methanol is widely studied due to its hole-scavenging ability. ${ }^{5-9}$ According to experimental data, the $\mathrm{O}-\mathrm{H}$ bond of methanol dissociates upon adsorption on the rutile $\mathrm{TiO}_{2}(110)$ surface with oxygen adatoms at $300 \mathrm{~K}$, yielding gaseous water and a pure layer of methoxy species residing on the five-fold-coordinated titanium rows. ${ }^{10}$ The methoxy intermediate is stable thermally, up to $600 \mathrm{~K}$, when thermal $\mathrm{C}-\mathrm{H}$ bond cleavage occurs, yielding formaldehyde and methanol. ${ }^{11}$ Cleavage of the methoxy $\mathrm{C}-\mathrm{H}$ bond with formation of formaldehyde can be alternatively achieved by creating an excitation across the titania band gap of 3.1 $\mathrm{eV} .^{1,8,12-15}$ The production of formaldehyde from methoxy on rutile $\mathrm{TiO}_{2}(110)$ is an excellent test case for simulating an elementary reaction driven by creation of an excited state. We used the self-consistent field $(\Delta-\mathrm{SCF})^{16}$ approach to create the photogenerated electron-hole pair. To capture the dynamics of the coupled electron-ion system, we used nonadiabatic Ehrenfest dynamics ${ }^{17,18}$ in the context of real-time timedependent density functional theory (TDDFT). This approach involves no empirical parameters and can reproduce with nearchemical accuracy the essential features of the system.

We modeled methanol photo-oxidation on rutile $\mathrm{TiO}_{2}(110)$ with methoxy bound to a five-fold-coordinated surface $\mathrm{Ti}$ and a $\mathrm{OH}$ at a neighboring bridge site from transfer of the methanol H. After geometry optimization, we sampled the potential energy surface using ground-state Born-Oppenheimer molecular dynamics (BOMD) at $200 \mathrm{~K}$ until thermal equilibration to obtain a variety of starting point configurations for excited-state dynamics, such as shown in Supporting Information Figure S1A. The majority of randomly selected configurations resulted

Received: February 27, 2015

Accepted: April 14, 2015 


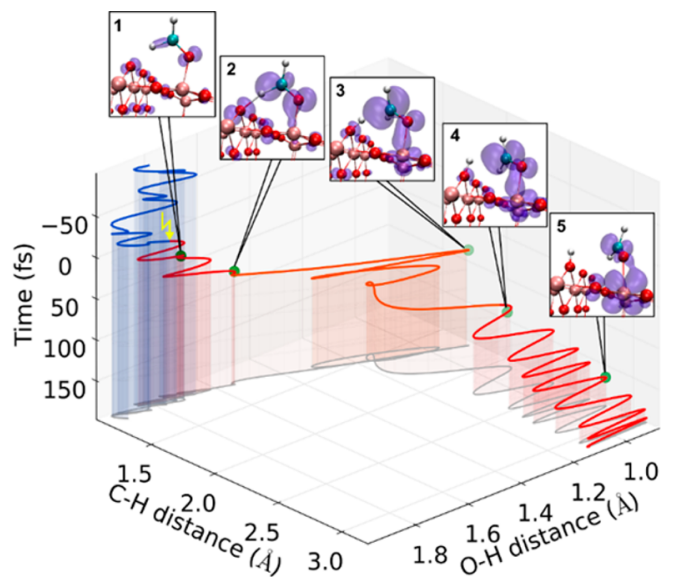

Figure 1. Methoxy to formaldehyde excited-state reaction trajectory: evolution of lengths of the $\mathrm{C}-\mathrm{H}$ bond being cleaved and the $\mathrm{O}-\mathrm{H}$ bond being formed. Reaction starts from methoxy on the upper left and proceeds to formaldehyde on the lower right. The blue curve corresponds to the ground-state molecular dynamics trajectory prior to the electronic excitation. The red curve corresponds to the excitedstate part of the trajectory. The bright red interval between frames 2 and 3 highlights part of the trajectory corresponding to rapid hydrogen transfer. The insets show snapshots of the hole orbital (blue cloud) at selected moments of time: 1-19.4, 2-53.2, 3-74.5, 4-118.0, and 5156.0 fs. On frames 1 and 2, this orbital represents depletion of spindown electrons from the $\mathrm{C}-\mathrm{H}$ bond and oxygen lone pairs, resulting in removal of the first electron from the adsorbate and formation of the formaldehyde anion radical with quenching of the hole; on frames $3-5$, it represents the transfer of an electron from the formaldehyde $\pi^{*}$ orbital to the surface titanium d orbital with formation of neutral formaldehyde and a polaron. in methoxy photo-oxidation during the first $200 \mathrm{fs}$ of the simulation, with all successful trajectories having a qualitatively similar reaction mechanism. In the following, we discuss one of the most illustrative trajectories in order to highlight the relevant features of the reaction mechanism. The excitation was created using the $\Delta$-SCF procedure that includes promotion of one electron from the highest occupied to the lowest unoccupied orbital and simultaneous relaxation of all orbitals, which resulted in an excitation energy of $2.5 \mathrm{eV}$. We tracked the time evolution of frontier orbitals, $\widetilde{\phi}_{N_{\text {occ }}}$ the "hole" state, from which electron is removed, and $\tilde{\phi}_{N_{\text {occ }}+1}$, the previously vacant "electron" state; jointly, those states represent the spin polarization in the system and highlight the chemically active areas. The evolution of the hole state is the most relevant for a photo-oxidation reaction; it represents depletion of spin-down electrons from previously occupied regions and accumulation of spin-up electrons in previously unoccupied regions (Movie 1, right panel). The response of the system to the motion of charge carriers can be inferred through changes of the key bond lengths, the $\mathrm{C}-\mathrm{H}$ bond being cleaved and the $\mathrm{O}-\mathrm{H}$ bond being formed, which are presented in Figure 1 along with the snapshots of the hole orbital. This plot shows the transition from the methoxy-like reagent region (upper left) to formaldehyde (lower right), with the $\mathrm{C}-\mathrm{H}$ bond cleavage occurring between 50 and 75 fs after photoexcitation (the transferred hydrogen atom will be denoted " $\mathrm{H}_{\mathrm{t}}$ " in the following discussion).

Remarkably, the movie of the hole evolution in real time contains a wealth of information about intermediate steps of the reaction; Movie 1 is presented as a web-enhanced object. This information is embodied in the shape changes of the hole orbital, which can be translated into a qualitative interpretation of the mechanism using Lewis structures; this is presented in
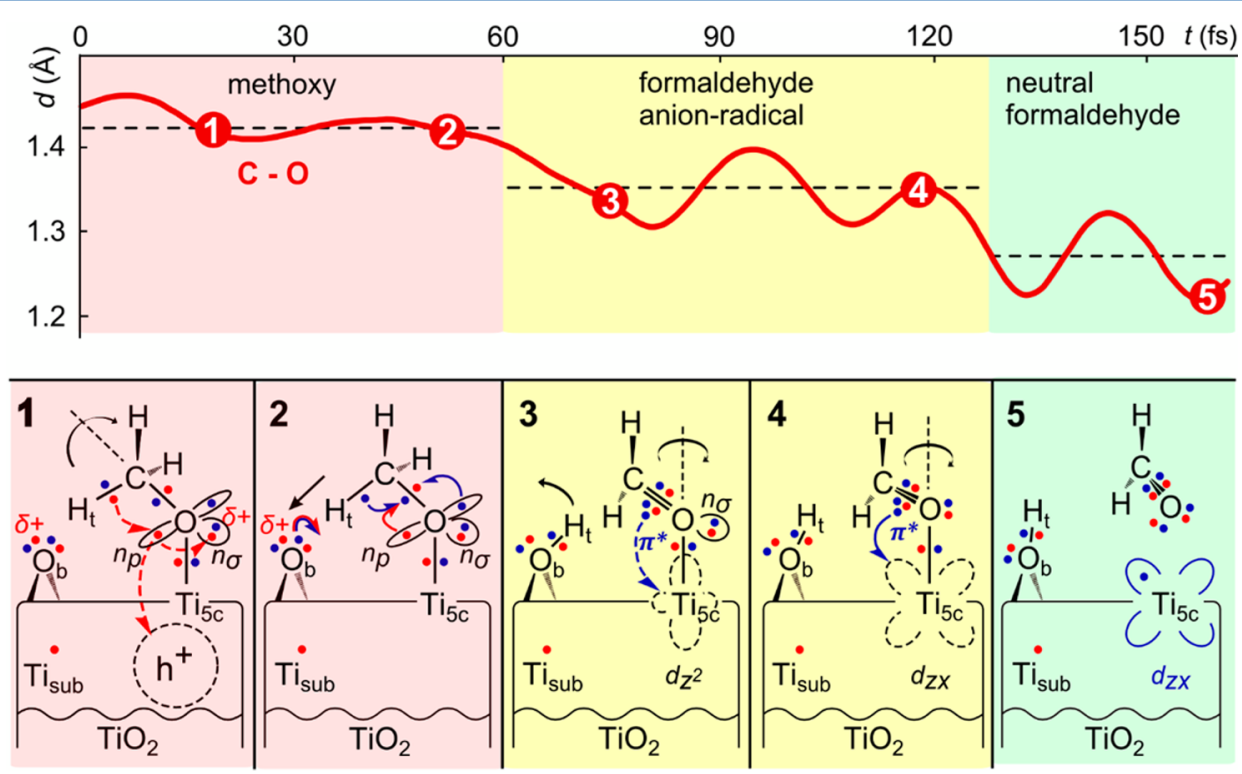

Figure 2. Photoinduced $\mathrm{C}-\mathrm{H}$ acidity oxidation mechanism. (Top) $\mathrm{C}-\mathrm{O}$ bond length in the adsorbate, with three regions corresponding to the qualitatively different electronic structure highlighted in different colors. Horizontal dashed lines represent the average $\mathrm{C}-\mathrm{O}$ bond length for each of the regions. (Bottom) Panels 1-5: Lewis structures indicating the rearrangement of the electronic structure during the reaction: colored circles, electrons of opposite spin; solid colored arrows, full electron transfer; dashed arrows, electron delocalization. Solid black arrows denote atomic motion, and dashed black lines denote rotational axes. Panel 1: the reorganization leading to complete hole transfer to the adsorbate; panel 2: heterolytic cleavage of the $\mathrm{C}-\mathrm{H}_{\mathrm{t}}$ bond and formation of adsorbate $\pi$ orbitals; panel 3: thermalization of the $\mathrm{O}-\mathrm{H}_{\mathrm{t}}$ bond and delocalization of an electron from the formaldehyde $\pi^{*}$ to surface titanium $\mathrm{d}_{z}{ }^{2}$; panel 4: rotation of the formaldehyde anion around the O-Ti axis facilitating complete electron transfer to the catalyst surface; panel 5: electronic structure of the products after reactive hole quenching. 

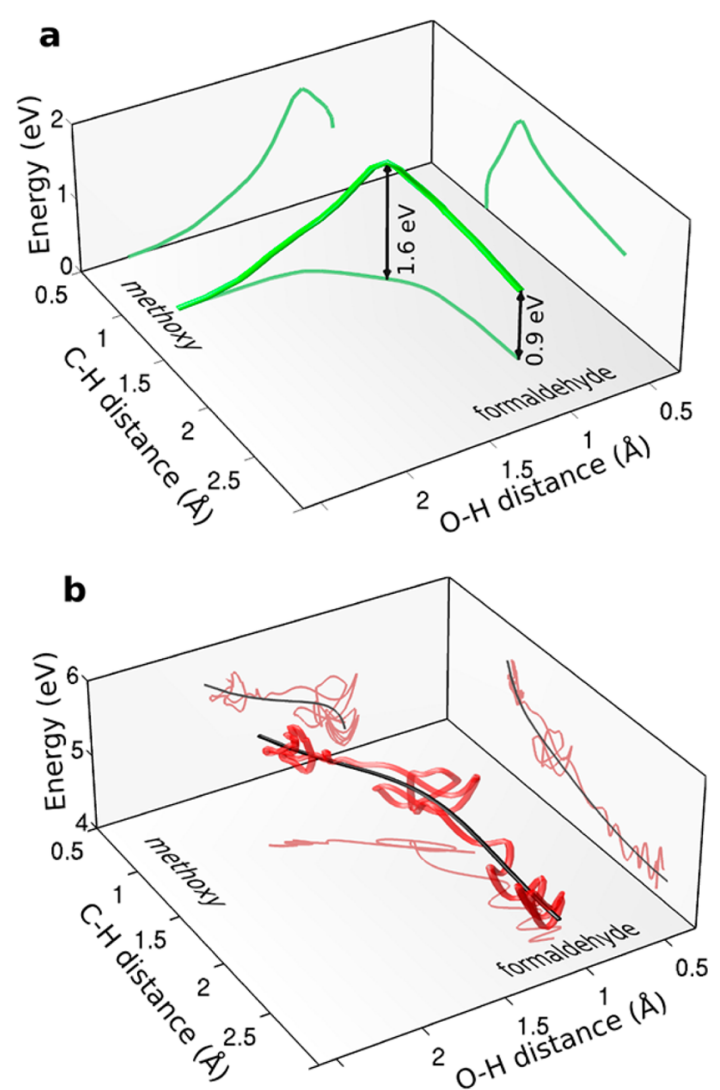

Figure 3. Energetics of the reaction. (a) NEB calculation (green) showing the $1.6 \mathrm{eV}$ barrier for the methoxy-to-formaldehyde reaction and the $0.9 \mathrm{eV}$ reaction enthalpy. (b) Electronic energy of the system after excitation along the reaction trajectory (red). The initial point of the trajectory is $5.5 \mathrm{eV}$ above the ground state. This includes 2.5 (the excitation energy) and $3 \mathrm{eV}$ (the thermal energy) of all unconstrained atoms in the simulation cell at $T=200 \mathrm{~K}$. The black line is guide to the eye. In the course of the reaction, the electronic system energy goes down by $1.2 \mathrm{eV}$.

Figure 2. After photoexcitation, the hole state is delocalized throughout the slab on p-type lone pairs of oxygen atoms and partly on the $\mathrm{n}_{\sigma}$ lone pair of methoxy. Contrary to the commonly held view, the hole is not "trapped" on any of the surface sites but is drawn to the molecule in the course of the reaction. The electron is transferred to the titanium atoms in the second subsurface layer with formation of a polaron (that is, an electron trapped in a lattice distortion), where it stays throughout the simulation trajectory (left panel of Movie 1 and Supporting Information Figure S2B). This behavior is consistent with previous work characterizing titanium $\mathrm{d}$ states as efficient electron traps. ${ }^{19,20}$

Initially, the hyperconjugation, ${ }^{21}$ that is, hybridization of the $\sigma_{\mathrm{C}-\mathrm{H}_{\mathrm{t}}}$ bond and $\mathrm{n}_{\sigma}$ lone pair due to their favorable coplanar arrangement, enables the electron transfer to the latter, which can be seen as spreading of hole on the $\mathrm{C}-\mathrm{H}_{\mathrm{t}}$ bond (frame 1 in Figures 1 and 2). Further, the hole moves to the less electronegative $\mathrm{p}$-rich $\mathrm{n}_{\mathrm{p}}$ lone pair of methoxy oxygen, and simultaneously, the $\mathrm{C}-\mathrm{H}_{\mathrm{t}}$ bond rotates to maintain the stabilizing hyperconjugative interaction. The more pronounced directional character of the $\mathrm{n}_{\mathrm{p}}$ pair compared to the s-rich $\mathrm{n}_{\sigma}$ enhances the $\mathrm{n}-\sigma$ hybridization and electron withdrawal from the $\mathrm{C}-\mathrm{H}_{\mathrm{t}}$ bond, resulting in complete one-electron oxidation of the adsorbate by $50 \mathrm{fs}$

$$
\mathrm{CH}_{3} \mathrm{O}_{\mathrm{ads}}^{-}+\mathrm{h}_{\mathrm{slab}}^{+}=\mathrm{CH}_{3} \mathrm{O}_{\mathrm{ads}}^{\bullet}
$$

Depletion of electron density from the $\mathrm{C}-\mathrm{H}_{\mathrm{t}}$ bond manifests itself as an increase of the hole lobe in that region (Figure 1 frame 2) and weakens the $\sigma_{\mathrm{C}-\mathrm{H}_{\mathrm{t}}}$ bond sufficiently to allow its pronouncedly heterolytic cleavage

$$
\mathrm{CH}_{3} \mathrm{O}_{\mathrm{ads}}^{\bullet}+\mathrm{O}_{\mathrm{b}}=\mathrm{CH}_{2} \mathrm{O}_{\mathrm{ads}}^{\bullet-}+\mathrm{OH}_{\mathrm{b}}^{+}
$$

Simultaneously with the $\mathrm{C}-\mathrm{H}$ acidic dissociation, the three electrons remaining on the former $\mathrm{C}-\mathrm{H}_{\mathrm{t}}$ bond and the oxygen $\mathrm{n}_{\mathrm{p}}$ hybridize to form the $\pi$ system of the formaldehyde anion radical

$$
\left(\sigma_{\mathrm{C}-\mathrm{H}_{\mathrm{t}}}\right)^{1+\delta}\left(\mathrm{n}_{\mathrm{p}, \mathrm{O}}\right)^{2-\delta} \rightarrow\left(\pi_{\mathrm{CH}_{2} \mathrm{O}}\right)^{2}\left(\pi_{\mathrm{CH}_{2} \mathrm{O}}^{*}\right)^{1}
$$

After this rearrangement, presented in Lewis notation in frame 2 of Figure 2, the hole corresponds to the singly occupied antibonding $\pi^{*}$ orbital of formaldehyde (Figure 1 frame 3 ). Accordingly, the $\mathrm{C}-\mathrm{O}$ bond length shortens to $1.35 \AA$ (Figure 2 middle panel, yellow highlighted region), a value between those typical of single and double bonds.

After a 40 fs thermalization period of the newly formed $\mathrm{O}-\mathrm{H}$ bond (Figure 1), resulting in complete decoupling of the $\mathrm{OH}_{b}$ and $\mathrm{CH}_{2} \mathrm{O}$ fragments, the system evolves to allow electron transfer from $\pi^{*}$ to titania. Initially, the formaldehyde anion radical $\pi^{*}$ orbital has overlap with the $\mathrm{d}_{z^{2}}$ orbital of the surface titanium atom, which can be seen as an extension of the hole lobe to the surface in frames 3 and 4 of Figure 1. However, it is hindered by symmetry, and the adsorbate rotates around the $\mathrm{O}-\mathrm{Ti}$ axis to achieve strong overlap with $\mathrm{Ti} \mathrm{d}_{z x}$, resulting in complete electron transfer to the surface with formation of a polaron, as shown in Figure 1 frame 5 and schematically represented in Figure 2 frame 4. Consistently with that, the average $\mathrm{C}-\mathrm{O}$ bond length changes to $1.23 \AA$, indicative of a double bond. The final stage of the process is another oneelectron oxidation step

$$
\mathrm{CH}_{2} \mathrm{O}_{\mathrm{ads}}^{\bullet-}=\mathrm{CH}_{2} \mathrm{O}_{\mathrm{ads}}+\mathrm{e}_{\mathrm{pol}}^{-}
$$

To illustrate the difference between the excited-state mechanism and a thermal reaction, we calculated the energy barrier using the climbing image nudged elastic band (CI-NEB) method. $^{22}$ The energy barrier for the reaction occurring with the electrons in their ground state at each step is $1.6 \mathrm{eV}$, and the reaction enthalpy is $0.9 \mathrm{eV}$ (Figure $3 \mathrm{a}$ ). On the excitedstate energy landscape, the system goes downhill in energy (Figure $3 \mathrm{~b}$ ), and no substantial energy barriers are encountered. The initial excitation energy of $2.5 \mathrm{eV}$ is expended in part (1.2 $\mathrm{eV}$ ) toward driving the endothermic methoxy-to-formaldehyde transformation, and the rest is dissipated through ionic motion. In the end of the excited-state trajectory, the electronic structure becomes qualitatively similar to that of the ground state of the products, namely, a neutral formaldehyde molecule and two bridging hydroxyls with the extra electrons localized as polarons on $\mathrm{Ti}$ atoms. Therefore, we can consider the adsorbate oxidation reaction as a pathway for excitation quenching.

Our analysis enables us to put forward general design principles for efficient photo-oxidation reactions. First is the strong coupling of the reactants and reactive intermediates to the semiconductor surface, in our case, methoxy and the formaldehyde anion. Second is the ability of the semiconductor surface to accommodate the reaction products; for the rutile $\mathrm{TiO}_{2}(110)$ surface, it is the presence of efficient electron- 
trapping sites on surface titanium atoms and Brønsted basic sites for proton abstraction on bridging oxygen atoms. Finally, the adsorbate should have nonhindered geometric and electronic structure rearrangement options, which make the hole on the molecule more favorable than being on the photocatalyst. In our case, this role was fulfilled by the hyperconjugation between the $\mathrm{C}-\mathrm{H}$ bond and the oxygen lone pairs, as well as sterically available rotations of different atomic groups in the adsorbate that sustained and enhanced that stabilizing effect. The above-mentioned properties will make the reaction pathway an efficient and fast channel for photogenerated carrier relaxation.

\section{ASSOCIATED CONTENT}

\section{S Supporting Information}

Methods, simulation slab setup (Figure S1), and snapshots of "electron" and "hole" wave function evolution (Figure S2). This material is available free of charge via the Internet at http:// pubs.acs.org.

\section{W Web-Enhanced Features}

Movie 1 of the non-adiabatic dynamics trajectory is available in the online version of the Letter. The green cloud in the left panel corresponds to the spin-down magnetization density of the "electron" state. The blue cloud in the right panel corresponds to the spin-up magnetization density or the "hole" state.

\section{AUTHOR INFORMATION}

\section{Corresponding Author}

*E-mail: kaxiras@physics.harvard.edu.

\section{Author Contributions}

${ }^{\perp}$ G.K. and D.V. contributed equally to this work.

\section{Notes}

The authors declare no competing financial interest.

\section{ACKNOWLEDGMENTS}

This work has been supported by National Science Foundation Catalaysis program under Grant Number CHE-1362616. The computations in this Letter were run partly on the Odyssey cluster supported by the FAS Division of Science, Research Computing Group at Harvard University. This work used the Extreme Science and Engineering Discovery Environment (XSEDE), which is supported by National Science Foundation Grant Number ACI-1053575.

\section{REFERENCES}

(1) Fujishima, A.; Zhang, X.; Tryk, D. A. $\mathrm{TiO}_{2}$ Photocatalysis and Related Surface Phenomena. Surf. Sci. Rep. 2008, 63, 515-582.

(2) Henderson, M. A. A Surface Science Perspective on $\mathrm{TiO}_{2}$ Photocatalysis. Surf. Sci. Rep. 2011, 66, 185-297.

(3) Kudo, A.; Miseki, Y. Heterogeneous Photocatalyst Materials for Water Splitting. Chem. Soc. Rev. 2009, 38, 253-278.

(4) Linsebigler, A. L.; Lu, G. Q.; Yates, J. T. Photocatalysis on $\mathrm{TiO}_{2}$ Surfaces - Principles, Mechanisms, and Selected Results. Chem. Rev. 1995, 95, 735-758.

(5) Migani, A.; Mowbray, D. J.; Iacomino, A.; Zhao, J.; Petek, H.; Rubio, A. Level Alignment of a Prototypical Photocatalytic System: Methanol on $\mathrm{TiO}_{2}$ (110). J. Am. Chem. Soc. 2013, 135, 11429-11432.

(6) Pang, C. L.; Lindsay, R.; Thornton, G. Chemical Reactions on Rutile $\mathrm{TiO}_{2}$ (110). Chem. Soc. Rev. 2008, 37, 2328-2353.

(7) Guo, Q.; Xu, C.; Yang, W.; Ren, Z.; Ma, Z.; Dai, D.; Minton, T. K.; Yang, X. Methyl Formate Production on $\mathrm{TiO}_{2}(110)$, Initiated by
Methanol Photocatalysis at $400 \mathrm{~nm}$. J. Phys. Chem. C 2013, 117, $5293-5300$

(8) Guo, Q.; Xu, C.; Ren, Z.; Yang, W.; Ma, Z.; Dai, D.; Fan, H.; Minton, T. K.; Yang, X. Stepwise Photocatalytic Dissociation of Methanol and Water on $\mathrm{TiO}_{2}(110)$. J. Am. Chem. Soc. 2012, 134, 13366-13373.

(9) Zhao, J.; Yang, J.; Petek, H. Theoretical Study of the Molecular and Electronic Structure of Methanol on a $\mathrm{TiO}_{2}(110)$ Surface. Phys. Rev. B 2009, 80, 235416.

(10) Phillips, K. R.; Jensen, S. C.; Baron, M.; Li, S.-C.; Friend, C. M. Sequential Photo-Oxidation of Methanol to Methyl Formate on $\mathrm{TiO}_{2}(110)$. J. Am. Chem. Soc. 2013, 135, 574-577.

(11) Farfan-Arribas, E.; Madix, R. J. Different Binding Sites for Methanol Dehydrogenation and Deoxygenation on Stoichiometric and Defective $\mathrm{TiO}_{2}(110)$ Surfaces. Surf. Sci. 2003, 544, 241-260.

(12) Shen, M.; Henderson, M. A. Identification of the Active Species in Photochemical Hole Scavenging Reactions of Methanol on $\mathrm{TiO}_{2} . J$. Phys. Chem. Lett. 2011, 2, 2707-2710.

(13) Shen, M.; Henderson, M. A. Role of Water in Methanol Photochemistry on Rutile $\mathrm{TiO}_{2}(110)$. J. Phys. Chem. C 2012, 116, 18788-18795

(14) Xu, C.; Yang, W.; Ren, Z.; Dai, D.; Guo, Q.; Minton, T. K.; Yang, X. Strong Photon Energy Dependence of the Photocatalytic Dissociation Rate of Methanol on $\mathrm{TiO}_{2}(110)$. J. Am. Chem. Soc. 2013, 135, 19039-19045.

(15) Shen, M.; Acharya, D. P.; Dohnalek, Z.; Henderson, M. A. Importance of Diffusion in Methanol Photochemistry on $\mathrm{TiO}_{2}(110)$. J. Phys. Chem. C 2012, 116, 25465-25469.

(16) Gorling, A. Density-Functional Theory beyond the Hohenberg-Kohn Theorem. Phys. Rev. A 1999, 59, 3359-3374.

(17) Meng, S.; Kaxiras, E. Real-Time, Local Basis-Set Implementation of Time-Dependent Density Functional Theory for Excited State Dynamics Simulations. J. Chem. Phys. 2008, 129, 054110.

(18) Tritsaris, G.; Vinichenko, D.; Kolesov, G.; Friend, C. M.; Kaxiras, E. Dynamics of the Photogenerated Hole at the Rutile $\mathrm{TiO}_{2}(110) /$ Water Interface: A Nonadiabatic Simulation Study. J. Phys. Chem. C 2014, 118, 27393-27401.

(19) Deskins, N. A.; Rousseau, R; Dupuis, M. Localized Electronic States from Surface Hydroxyls and Polarons in $\mathrm{TiO}_{2}(110)$. J. Phys. Chem. C 2009, 113, 14583-14586.

(20) Kowalski, P. M.; Camellone, M. F.; Nair, N. N.; Meyer, B.; Marx, D. Charge Localization Dynamics Induced by Oxygen Vacancies on the $\mathrm{TiO}_{2}(110)$ Surface. Phys. Rev. Lett. 2010, 105, 146405.

(21) Weinhold, F.; Landis, K. Valency and Bonding: a Natural Bond Orbital Donor-Acceptor Perspective; Cambridge University Press: New York, 2005; pp 215-275.

(22) Henkelman, G.; Uberuaga, B. P.; Jonsson, H. A Climbing Image Nudged Elastic Band Method for Finding Saddle Points and Minimum Energy Paths. J. Chem. Phys. 2000, 113, 9901-9904. 